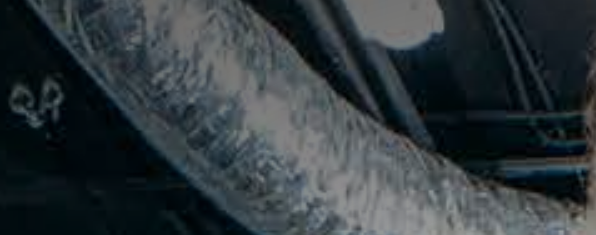

Artículo Invitado / Invited Article

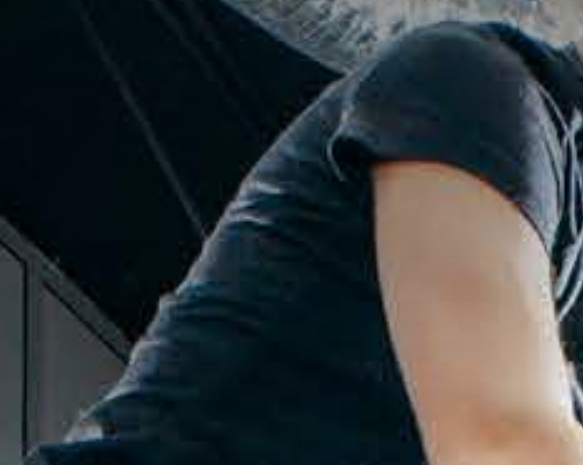

\title{
Efectos de un
}

entranamiento físico

para estudiantes de

Did $x^{2}=$

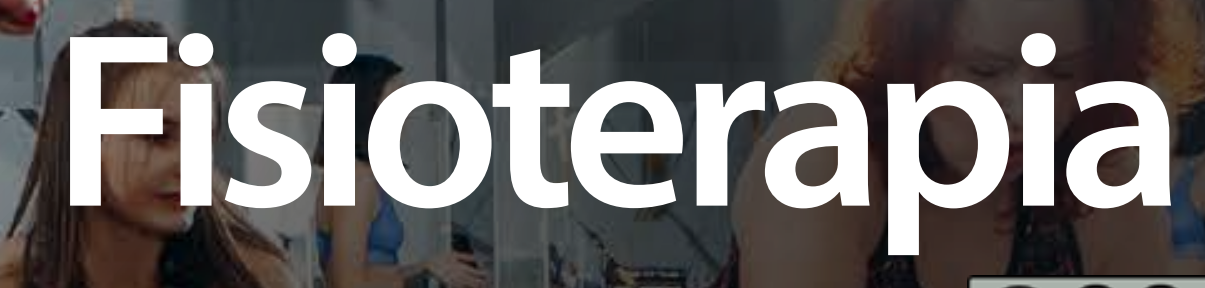

Effects of a training program on physical therapy students

(c) $\frac{10(2)(2)}{{ }_{\mathrm{BY}} \mathrm{NC} \text { SA }}$

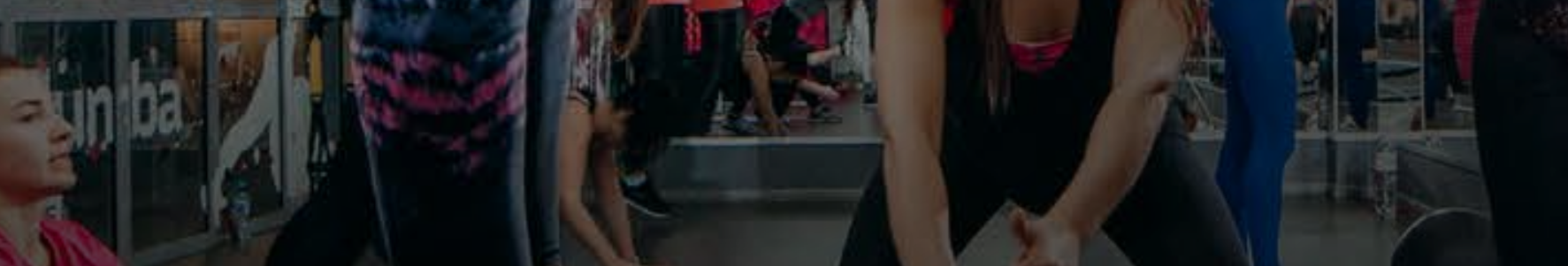

Claudia Ximena Molina-Arellano

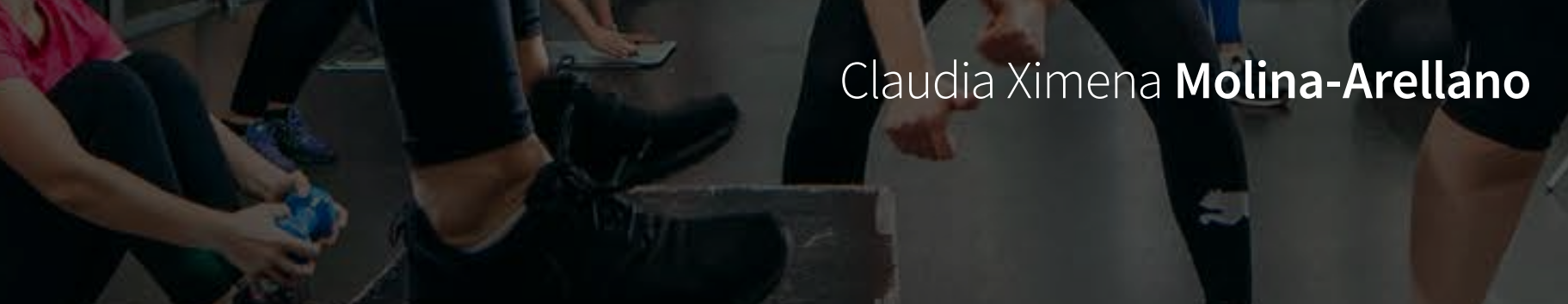

\section{Movimiento cientifico}

ISSN-l: 2011-7191 | e-ISSN: 2463-2236 
Movimiento Cientifico

ISSN-l: 2011-7191 | e-ISSN: 2463-2236 Publicación Semestral

ID: 2011-7191.mct.13202

Title: $\quad$ Effects of a training program on physical therapy students

Título: Efectos de un programa de entrenamiento físico para estudiantes de Fisioterapia

\section{Alt Title / Título alternativo:}

[en]: Effects of a training program on physical therapy students

[es]: $\quad$ Efectos de un programa de entrenamiento físico para estudiantes de Fisioterapia

Author (s) / Autor (es):

Molina-Arellano

Keywords / Palabras Clave:

[en]: $\quad$ Physical Education and Training; Students; Physical Therapy

[es]: $\quad$ Educación y entrenamiento físico; estudiantes; Fisioterapia

Submited: $\quad$ 2019-06-28

Acepted: $\quad$ 2019-09-25

\section{Resumen}

Introducción: la práctica de actividad física en los estudiantes y profesionales en fisioterapia a nivel nacional es deficiente, aumentando los niveles mundiales de sedentarismo, por lo que se propuso un programa de entrenamiento validado para entrenamiento físico de futuros profesionales en fisioterapia que mejoren las cualidades físicas más importantes para el desempeño profesional.

Objetivos: EL objetivo de esta investigación fue determinar los efectos de un programa de entrenamiento en las cualidades físicas de los estudiantes de VIII y IX semestre del programa de Fisioterapia. Materiales y métodos: Se convocó a 46 participantes estudiantes de VIII y IX semestre del programa de Fisioterapia matriculados, aptos para el programa de entrenamiento, se realizó la aleatorización para determinar los grupos control y experimental con 23 participantes por cada grupo, se midieron las cualidades físicas pre y post programa con entrenamiento de 12 semanas. Como pruebas estadísticas se aplicó t de Student y Mann-Whitney, para determinar el tamaño del efecto del programa se aplicó d de Cohen. Resultados: En todas las cualidades físicas se encontraron cambios estadísticamente significativos ( $P$ valor $<$ al 0.05), con efectos relevantes según el estadístico $d$ de Cohen ( $d>$ al 0.2) debido al programa de entrenamiento físico en resistencia cardiovascular, fuerza y flexibilidad. Conclusiones: El programa de entrenamiento mejoró todas las cualidades físicas utilizando método de entrenamiento funcional, autor cargas y flexibilidad global y dinámica y es aplicable a poblaciones similares.

\section{Abstract}

Background: Practice of physical activity by students and professionals in

physiotherapy is deficient in Colombia increasing the global levels of sedentary lifestyle. A validated program for physical training of future professionals in physiotherapy that improve the most important physical qualities was proposed. for professional performance. Objectives: The objective of this research was to determine the effects of a training program on the physical qualities of the students of VIII and IX semester of the

Physiotherapy program. Materials and methods: 46 participants of VIII and IX semester students of the Physiotherapy program enrolled, eligible for the training program, were invited to randomize the control and experimental groups with 23 participants for each group, the qualities

were measured physical pre and post program with a 12 week training program. As statistical tests T- Student and MannWhitney were applied, to determine the size of the program effect was applied by $d$ Cohen. Results: Statistically significant changes were found in all physical qualities ( $P$ value $<0.05$ ), with relevant effects according to the $d$ Cohen statistic (d> at 0.2) due to the physical training program in cardiovascular resistance, strength and flexibility. Conclusions: The training program improved all physical qualities using functional training method, self-loads and global and dynamic flexibility and is applicable to similar populations.

\section{Citar como:}

Molina-Arellano, C. X. (2019). Efectos de un programa de entrenamiento físico para estudiantes de Fisioterapia. Revista Iberoamericana de Psicología issn-l:2011-7191, 13 (2), 37-46. Obtenido de: https://revmovimientocientifico.ibero.edu.co/article/view/1500

$\mathrm{BIO}$ :

Docente Programa de Fisioterapia; Universidad Mariana

City | Ciudad:

Pasto [co]

e-mail:

cmolina@umariana.edu.co 


\section{Efectos de un programa de entrenamiento físico para estudiantes de Fisioterapia}

Effects of a training program on physical therapy students Claudia Ximena Molina-Arellano

\section{Introducción}

El marco de la investigación tiene como actor principal el profesional y estudiante de Fisioterapia. Para identificar el problema y el vacío en el conocimiento a nivel nacional en diferentes programas de fisioterapia, se tuvo en cuenta la ley que rige y define esta profesión en Colombia 528 de 1999, especialmente por su definición que describe: "la Fisioterapia es la profesión liberal del área de la salud que contribuye al desarrollo humano y social de los individuos y colectivos humanos con los cuales trabaja, orienta sus acciones al mantenimiento, optimización o potencialización del movimiento, así como a la prevención y recuperación de sus alteraciones"(Legislativa 1999), entonces el profesional en Fisioterapia forma parte fundamental en todas las empresas de servicios de salud y en equipos de trabajo interdisciplinario, ya que su campo de desempeño es amplio, e incluye acciones de intervención que requiere habilidades específicas del fisioterapeuta, entre estas las que implican orientación y ejecución de ejercicios terapéuticos, movilización de segmentos corporales, movilidad de pacientes, ejecución de técnicas manuales entre otras, que sin una adecuada preparación física como fuerza, flexibilidad y resistencia cardiovascular, no son efectivas y podrían generar lesiones a los profesionales en un trabajo repetitivo y de alta carga como son los servicios de Fisioterapia del país. Se encontró en un estudio que la Fisioterapia es una profesión de alto impacto a nivel mundial debido a su aporte a desarrollo económico y social por la recuperación funcional de las personas (Mahecha, 2012). Algunos fisioterapeutas que son activos y lo refieren en diversos estudios, podrían tener algunas ventajas en mantenimiento y mejora de su salud, sin embargo, según McPhail y Waite, las estrategias de acondicionamiento físico que practican los Fisioterapeutas no son útiles para la prevención de desórdenes musculoesqueléticos y se indicó crear propuestas de acondicionamiento específicas ( (Mcphail, 2014)). Por este aporte valioso de información se diseñó un programa específico a la población estudiantil en preparación para la vida laboral del Fisioterapeuta que permita potenciar la fuerza, flexibilidad y resistencia cardiovascular, importantes para su actividad laboral y su salud física. 
Sedentarias para lograr un tiempo de vida largo que maximice el bienestar desde el ámbito universitario. ( (Peterson, 2018)) .

Las universidades deben formar un papel fundamental en la práctica de actividad física y hábitos de vida saludable, ya que esto debe hacerse desde la Universidad Saludable, proyecto que se presentó en la Conferencia "Construyendo universidades Saludables " en Santiago de Chile en 2003 bajo el auspicio de la Organización Panamericana de la Salud (OPS) y se concluyó que este programa debe centrar esfuerzos para promoción de hábitos de vida saludables y debe construir espacios para promover la salud (chaparro, 2014) En el caso de la Universidad Mariana, en la ciudad de Pasto, se cuenta con el curso de desarrollo humano y equipos deportivos donde hay un mínimo número de estudiantes del programa de Fisioterapia participantes de estas actividades, razón por la cual se ha identificado una deficiencia en las cualidades físicas teniendo en cuenta el desempeño físico que esta profesión requiere durante las prácticas formativas; por ello se propuso diseñar y validar un programa de entrenamiento físico para las cualidades físicas: resistencia cardiovascular, flexibilidad y la fuerza muscular y que se implemente dentro del pensum académico como parte de su formación, siendo un espacio de bienestar, promocionando la salud y el ejercicio y facilitando el desarrollo de las cualidades físicas básicas del fitness para mejorar el desempeño estudiantil y profesional.

Los estudiantes de la Universidad Mariana, en el Programa de Fisioterapia, no contaban con un programa de entrenamiento físico para prevenir el sedentarismo y no existe una propuesta o guía de ejercicios recomendados para los requerimientos propios de estudiantes y profesionales en Fisioterapia con fines de prevención de lesiones, mejoría y mantenimiento de su salud, siendo los resultados de esta investigación un punto de partida fundamental para todas las universidades quienes estén interesadas en lograr fisioterapeutas con capacidades físicas que permitan un mejor desempeño educativo y laboral. Esto lograría prevenir lesiones tempranas e incapacitantes, ya que existe importante evidencia de la relación entre desordenes musculo esqueléticos y la actividad laboral de Fisioterapeutas con una prevalencia del 90\%, mientras el 50\% de estos profesionales experimenta estas alteraciones en los primeros 5 años por actividades repetitivas, posturas prolongadas, movimientos de flexo extensión y rotación continua (Anderson \& Oakman, 2016), y otro estudio demostró que los fisioterapeutas que más se afectaron de alteraciones musculo esqueléticas trabajaron entre 8 a 9 horas en centros de rehabilitación y hospitales( (BAE, Young-Hyeon, and Kyoung Sam MIN. , 2016)

Aunque en los últimos años, se ha incrementado la cultura de la práctica de ejercicio, y del pensamiento "saludable," los jóvenes y especialmente los universitarios, parecen los menos interesados en estas opciones, presentando diferentes problemas de salud que afectan toda la esfera biopsicosocial, conociendo que sus principales afecciones incluyen problemas de salud mental (incluyendo la ansiedad y la autolesión), el abuso de sustancias, alteraciones del sueño y mala nutrición (Hagell, 2013).

El programa de entrenamiento que se validó y aplicó en esta investigación, facilitó desviar esos aspectos negativos del sedentarismo o el mal uso del tiempo libre, a tiempo valioso de entrenamiento, práctica de ejercicio, y cuidado de la salud para los futuros Fisioterapeutas. Para la comunidad científica universitaria, el aporte de esta investigación y el cumplimiento de sus objetivos permitieron demostrar que el entrenamiento planificado y dirigido logra cambios importantes en las cualidades físicas y en la salud de las personas.

\section{Materiales y métodos:}

La presente investigación se enmarcó dentro del enfoque empírico analítico, paradigma cuantitativo y tipo experimental con grupo control. Se identificaron tres objetivos, que consistieron en hacer diseño y validación de un programa de entrenamiento físico para estudiantes de VIII y IX semestre del programa de Fisioterapia, medición de las cualidades físicas pre y post programa de entrenamiento y la comparación de los efectos del programa en las capacidades físicas post intervención del grupo control con el grupo experimental. En el marco ético y legal se utilizó la Resolución 8430 del 4 de octubre de 1993, para el desarrollo de la investigación que se fundamentó en la intervención con seres humanos, el Artículo 11 que clasificó esta investigación como riesgo mínimo y el Artículo 25, por el cual se diligenció el consentimiento informado, en el cual se explicó cada uno de los aspectos contenidos en el mismo y de la misma manera se respetó la confidencialidad en el desarrollo de la investigación. El consentimiento informado fue verificado y validado por el Comité de Ética de la Universidad Mariana y se aplicaron los cuatro principios éticos básicos: respeto por las personas, beneficencia, no maleficencia y justicia.

El proceso para los grupos control y experimental inició con una cita para los estudiantes matriculados del programa de Fisioterapia de la Universidad Mariana en los semestres VIII y IX del año 2015, con un total de 54 estudiantes a quienes se les aplicó un test para el ejercicio físico, donde se identificaron contraindicaciones absolutas o aptitud para la práctica de ejercicio. Los 46 estudiantes aptos entraron a un proceso de aleatorización utilizando Microsoft Excel 2010, con una macro validada por Visual Basic. Se obtuvo 2 grupos de 23 participantes, para grupo control y experimental. Se postuló como hipótesis nula: Posterior a la aplicación del programa de entrenamiento, las cualidades físicas de los estudiantes del grupo experimental, no mejoran con relación a las del grupo control.

El programa de entrenamiento, luego de su validación por expertos a nivel nacional, el cual utilizó el método de periodización del entrenamiento, por medio del diseño de un macrociclo, microciclos, periodos, sesiones y actividades, en donde se planificó diferentes intensidades y métodos de entrenamiento funcional (suspensión, circuitos interválicos, autocargas y fortalecimiento funcional), teniendo en cuenta la población "sana" sedentaria y las edades promedio del grupo experimental. Fue aplicado por un total de 12 semanas al grupo experimental, mientras el grupo control asistió al mismo número de sesiones a capacitación en promoción de la salud y prevención de la enfermedad por medio del ejercicio. Se aplicó la investigación con un método doble ciego, investigadores modalidad III, y la participación de estudiantes del programa en coinvestigación quienes aportaron con el segundo objetivo de la investigación. Dos grupos diferentes evaluaron las cualidades físicas pre y post entrenamiento mientras los docentes investigadores aplicaron el programa.

La resistencia cardiovascular y V02máximo se midió con el Test de Cooper, la fuerza muscular con el test de 1 RM para miembros superiores e inferiores, para la flexibilidad de cadena muscular posterior se utilizó el test de Sit and Reach y para identificar el rango de movimiento de la cadera derecha e izquierda se utilizó el test de goniometría. El test de Cooper, permitió evaluar el grupo en una sola sesión tanto en pretest como en postest con un mínimo de tiempo y logística requerido para la actividad. El test de fuerza se realizó en banco para pectorales y en press de pierna para miembros inferiores siendo ejecuciones sencillas de bajo riesgo de lesiones. 
El programa de entrenamiento se aplicó 3 veces por semana, con una duración entre 90 a 120 minutos, se utilizó la metodología de la periodización con tres periodos, preparatorio general, preparatorio específico y de mantenimiento o recuperador. Finalizó el programa un grupo de 10 estudiantes para el grupo experimental y un total de 7 para el grupo control. Algunos estudiantes se retiraron por entrar en periodo de vacaciones durante la aplicación del programa de entrenamiento y otros por inasistencia a las sesiones. El progra- ma de entrenamiento físico se fundamentó en las tendencias recomendadas por la American College of Sports Medicine, publicadas cada año, en este caso en el año 2014, donde se encontró el entrenamiento interválico de alta intensidad, el ejercicio con el propio peso corporal y el circuito funcional. Se utilizó elementos como suspensión, balones medicinales, pesos libres, fit balls, bases inestables, resistencias elásticas y campo libre.

Figura 1 Modelo del macrociclo de entrenamiento diseñado y validado.

\begin{tabular}{|c|c|c|c|c|c|c|c|c|c|c|c|c|c|c|c|c|c|c|c|c|c|}
\hline \multicolumn{22}{|c|}{ INVESTIGACÓN PROFESORAL: Efectos de un programa de entren } \\
\hline INVESTIGADORE & Esp. Claudia Ximena N & Molina & A. -.- & ic. Ft. $H$ & Manu & & le Sala & & UNIVE & $\overline{S I D A D}$ & MAR & NA, & ACULT & $\overline{\mathrm{DCl}}$ & IENCIA & ASDE L & A SALUI & JD, PROG & AA DE FIS & OTERA & \\
\hline \multicolumn{2}{|c|}{ MACROCICLO } & \multicolumn{20}{|c|}{1} \\
\hline \multicolumn{2}{|c|}{ MESES } & \multicolumn{4}{|c|}{ MARZO } & \multicolumn{3}{|r|}{ ABRIL } & & & \multicolumn{5}{|c|}{$\frac{3}{\text { MAYO }}$} & & & JUNIO & & & \\
\hline \multicolumn{2}{|c|}{ PERIODO } & \multicolumn{2}{|c|}{ Evaluación } & \multicolumn{5}{|c|}{ PREPARATORIO GENERAL - ADAPTACIÓN } & \multicolumn{9}{|c|}{ PREPARATORIO ESPECIFICO -ACONDICIONAMIENTO } & \multicolumn{2}{|c|}{ RECUPERADOR } & \multicolumn{2}{|c|}{$\begin{array}{c}\text { Evaluación } \\
\text { final }\end{array}$} \\
\hline \multicolumn{2}{|c|}{ MICROCICLOS } & 1 & 2 & 3 & 4 & & 5 & 6 & 7 & 8 & 9 & 10 & 11 & & 12 & 13 & 14 & 15 & 16 & 17 & 18 \\
\hline \multicolumn{2}{|c|}{ TIPO MICROCICLO } & & & \multicolumn{5}{|c|}{ GRADUAL } & \multicolumn{9}{|c|}{ DE CHOQUE } & DE RECl & RACIÓN & & \\
\hline SESIONES PC & MICROCICLO & 3 & 3 & 3 & 3 & & 3 & 3 & 3 & 3 & 3 & 3 & 3 & & 3 & 3 & 3 & 2 & 2 & 3 & 3 \\
\hline HORAS & POR DIA & 2 & 2 & 1 & 1 & 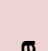 & 1 & 1 & 1 & 1 & 1 & 1 & 1 & & 1 & 1 & 1 & 1 & 1 & 2 & 2 \\
\hline & TEST & & & & & $\stackrel{\Perp}{\rightleftarrows}$ & & & & & & & & & & & & & & & \\
\hline & TEST & & & & & 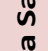 & & & & & & & & & & & & & & & \\
\hline PORC & NTAJE & & & $50 \%$ & & & $0 \%$ & & & & & & $30 \%$ & & & & & & & & \\
\hline VOLUMENTO T & TALEN MINUTOS & $360^{\prime}$ & $360^{\prime}$ & 240 & 240 & & 240 & $240^{\prime}$ & 270 & 270 & 270 & 27 & 27 & & 270 & 270 & 270 & $120^{\prime}$ & $120^{\prime}$ & $360^{\prime}$ & $360^{\prime}$ \\
\hline COMPONEN & S DEL FITNESS & 720 & $0^{\prime}$ & & & 960 & & & & & & & $160^{\prime}$ & & & & & & & & $0^{\prime}$ \\
\hline RESISTENCI & CARDIORESPIRATORIA & & & $40 \%$ & $30 \%$ & & $40 \%$ & $30 \%$ & $30 \%$ & $30 \%$ & $40 \%$ & $25 \%$ & 259 & & $0 \%$ & $25 \%$ & $35 \%$ & $35 \%$ & $35 \%$ & & \\
\hline Prep. Fisica general. & Circuito funcional & & & $96^{\prime}$ & 72 & & $96^{\prime}$ & $72^{\prime}$ & $81^{\prime}$ & $81^{\prime}$ & 108 & 67. & 67. & & $135^{\prime}$ & 67.5 & $94.5^{\prime}$ & $42^{\prime}$ & $42^{\prime}$ & & \\
\hline Resistencia aerobica & Vuelta a la calma & & & & & 茎 & & & & & & & & & & & & & & & \\
\hline FUERZA & IUSCULAR & & & $40 \%$ & $40 \%$ & $n_{0}^{\circ}$ & $40 \%$ & $40 \%$ & $40 \%$ & $30 \%$ & $30 \%$ & 50 & $25 \%$ & & $5 \%$ & $50 \%$ & $30 \%$ & $35 \%$ & $35 \%$ & & \\
\hline Circuito funcional & Fuerza funcional & & & $96^{\prime}$ & $96^{\prime}$ & $\frac{5}{\pi}$ & $96^{\prime}$ & $96^{\prime}$ & $108^{\prime}$ & $81^{\prime}$ & $81^{\prime}$ & 135 & 67. & & 77.5 & $135^{\prime}$ & $81^{\prime}$ & $42^{\prime}$ & $42^{\prime}$ & & \\
\hline Resistencia aerobica & & & & & & $\frac{\xi}{0}$ & & & & & & & & & & & & & & & \\
\hline FLEXI & ILIDAD & & & $20 \%$ & $30 \%$ & & $20 \%$ & $30 \%$ & $30 \%$ & $40 \%$ & $30 \%$ & $25 \%$ & $50 \%$ & & $25 \%$ & $25 \%$ & $35 \%$ & $30 \%$ & $30 \%$ & & \\
\hline
\end{tabular}

Fuente: elaboración propia (2017)

\section{Análisis de resultados:}

Para identificar el nivel de las cualidades físicas pre y post aplicación del programa de entrenamiento en grupo control y experimental, se hizo una asignación aleatorizada. Se midió cada cualidad física, pre y post aplicación del programa de entrenamiento, se aplicaron las pruebas de normalidad de Shapiro Wilk y de homogeneidad de varianzas de Levenne pre y post, con el fin de definir las pruebas estadísticas apropiadas para establecer las diferencias entre grupo experimental y control en pre y post aplicación del programa.

Se determinó las diferencias entre grupo control y experimental de las diferencias entre pre y post programa (diferencias en diferencias DID), para establecer el efecto del programa.

Para determinar si las (DID) fueron significativamente estadísticas se utilizó la prueba t de student.
Cuando se observó normalidad en los datos y la prueba de Mann- Whitney cuando no la hubo.

Para determinar el tamaño del efecto del programa se utilizó el estadístico d de Cohen (DID estandarizadas), con la siguiente escala: propuesta por Cohen (1998): si d está en [0.0, 0.2] la DID es trivial o muy pequeña, si está en $(0.2,0.5]$ es pequeña, en $(0.5,0.8]$ es moderada y (0.8, infinito) es grande. 7 Siendo relevantes las diferencias si el valor esta encima de 0.2 .

\section{Resultados:}

Para el primer objetivo, se realizó un proceso de búsqueda de información para documentación teórica sobre metodología del entrenamiento, fisiología del entrenamiento y programación del ejercicio para la juventud. Después de crear el plan de entrenamiento y dise- 
ñar el macrociclo con las respectivas sesiones, se envió a expertos para su validación. Se tuvo en cuenta la documentación aportada de la American College of Sports Medicine, así como Journal of Medicine \& Science in Sports \& Exercise ${ }^{\circledR}$, y los fundamentos de prescripción de ejercicio de Vivian Heyward. Los expertos validaron el programa de entrenamiento con un puntaje promedio de 50 puntos, después de algunos cambios recomendados a algunos ejercicios para la población juvenil sedentaria disminuyendo cargas y dificultad y posteriormente se llevó a cabo la aplicación del programa.

Tabla 1. Tabla resumen de las diferencias de cualidades físicas pre y post programa entre los grupos control y experimental (DID), p valor y d Cohen.

\begin{tabular}{|c|c|c|c|c|c|c|c|c|c|}
\hline \multirow{2}{*}{ Cualidad Física } & \multicolumn{3}{|c|}{ G. Experimental } & \multicolumn{3}{|c|}{ G. Control } & \multirow{2}{*}{$\mathrm{DID}^{*}$} & \multirow{2}{*}{$\begin{array}{l}\text { pvalor del } \\
\text { DID }\end{array}$} & \multirow{2}{*}{ D Cohen } \\
\hline & Pre & Post & Dif & Pre & Post & Dif. & & & \\
\hline $\begin{array}{c}\text { Flexibilidad cadena muscular } \\
\text { posterior (Sit and Reach) }\end{array}$ & $-1,4$ & 7,11 & 8,51 & 1 & 0,14 & $-0,86$ & 9,37 & $0,000 b\left({ }^{\star *}, b\right)$ & 2,38 \\
\hline $\begin{array}{c}\text { Movilidad (Flexión Cadera } \\
\text { Derecha) }\end{array}$ & 82,7 & 107,1 & 24,4 & 81,71 & 82,43 & 0,71 & 23,69 & $0,000\left({ }^{* *} \mathrm{C}\right)$ & 3,41 \\
\hline $\begin{array}{c}\text { Movilidad (Flexión Cadera } \\
\text { Izquierda) }\end{array}$ & 89 & 105,8 & 16,8 & 84,57 & 84,14 & $-0,43$ & 17,23 & $0,009\left({ }^{* *} \mathrm{C}\right)$ & 1,34 \\
\hline $\begin{array}{c}\text { Movilidad Extensión Cadera } \\
\text { Derecha }\end{array}$ & 14,8 & 22,5 & 7,7 & 13,57 & 15 & 1,43 & 6,27 & $0,000\left(^{* \star b}\right)$ & 2,13 \\
\hline $\begin{array}{c}\text { Movilidad (Extensión Cadera } \\
\text { Izquierda) }\end{array}$ & 14,9 & 22,3 & 7,4 & 14,71 & 15 & 0,29 & 7,11 & $0,000\left(^{* *} \mathrm{~b}\right)$ & 3,4 \\
\hline $\begin{array}{c}\text { Movilidad (Abducción Cadera } \\
\text { Derecha) }\end{array}$ & 33,1 & 61,7 & 28,6 & 40,57 & 40,43 & $-0,14$ & 28,74 & $0,000\left({ }^{\star \star} \mathrm{C}\right)$ & 2,31 \\
\hline $\begin{array}{l}\text { Movilidad (abducción cadera } \\
\text { izquierda) }\end{array}$ & 32,8 & 59,5 & 26,7 & 39,86 & 42 & 2,14 & 24,56 & $0,001\left({ }^{\star *} \mathrm{C}\right)$ & 1,97 \\
\hline $\begin{array}{l}\text { FUERZA MUSCULAR (Fases del } \\
\text { CORE) }\end{array}$ & 2,9 & 5,1 & 2,2 & 3,43 & 3,14 & $-0,29$ & 2,49 & $0,002\left({ }^{* *} \mathrm{C}\right)$ & 1,79 \\
\hline $\begin{array}{l}\text { FUERZA MAXIMA MIEMBROS } \\
\text { SUPERIORES IRM }\end{array}$ & 29 & 31 & 2 & 22,14 & 21,43 & $-0,71$ & 2,71 & $0,01\left({ }^{\star \star} \mathrm{C}\right)$ & 1,94 \\
\hline $\begin{array}{l}\text { FUERZA MAXIMA MIEMBROS } \\
\text { INFERIORES } 1 \text { RM }\end{array}$ & 118,6 & 124,5 & 5,9 & 118,57 & 56,86 & $-61,71$ & 67,61 & $0,010\left(^{* \star}\right) c$ & 2,22 \\
\hline
\end{tabular}

Fuente: elaboration propia (2017)

Dif: post test menos pre test

DID: dif grupo control menos dif grupo experimental.

P valor * altamente significativos ( $p$ valor $<0.001$ )

b Prueba T asumiendo varianzas diferentes

c prueba Mann-Whitney por falta de normalidad.

La tabla 1 indicó la medición pre test y post test de las cualidades físicas en grupo experimental y control. Para el grupo experimental se evidencia la mejoría en todas las cualidades físicas, siendo de mayor diferencia la flexibilidad de cadena muscular posterior con un valor negativo en el pre test, para mejorar hasta $7.11 \mathrm{~cm}$ después del entrenamiento. La flexión de cadera derecha por ejemplo, mejoró en $24.4^{\circ}$, la fuerza Core que inició en promedio de 2.9 fases mejoró a 5 fases alcanzadas y el VO2 Máximo en el test de Cooper, indicó un aumento de 635.3 metros recorridos en los participantes del grupo experimental. El grupo control, por el contrario, según la tabla, mostró diferencias (Dif.) negativas como el caso la flexibilidad de músculos posteriores, la flexión de cadera izquierda, la abducción de cadera derecha, la resistencia cardiovascular, e incluso la fuerza de miembros superiores e inferiores, demostrando una disminución de las capacidades físicas sin el programa de entrenamiento físico. En el grupo experimental, los resultados fueron significativamente estadísticos, con una magnitud del cambio según d de Cohen grande y el $p$ valor con mediciones menores al 0.05, $(P<0,02)$, demostrando que el programa de entrenamiento se pude extrapolar a poblaciones similares y el programa tuvo alta efectividad para esta población, logrando que las cualidades físicas mejoren y se potencien. 


$\begin{array}{ll}\text { Stan Reach } & 3,40 \\ \text { Flexión Cadera Derecha } & 4,90 \\ \text { Flexión Cadera Izquierda } & 1,80 \\ \text { Extensión Cadera Derecha } & 3,10 \\ \text { Extensión Cadera Izquierda } & 4,90 \\ \text { Abducción Cadera Derecha } & 3,30 \\ \text { Abducción Cadera Izquierda } & 2,40 \\ \text { Fases Core } & 2,30 \\ \text { Test Cooper } & 5,00 \\ \text { RMMS } & 2,90 \\ \text { RMM } & 3,20\end{array}$

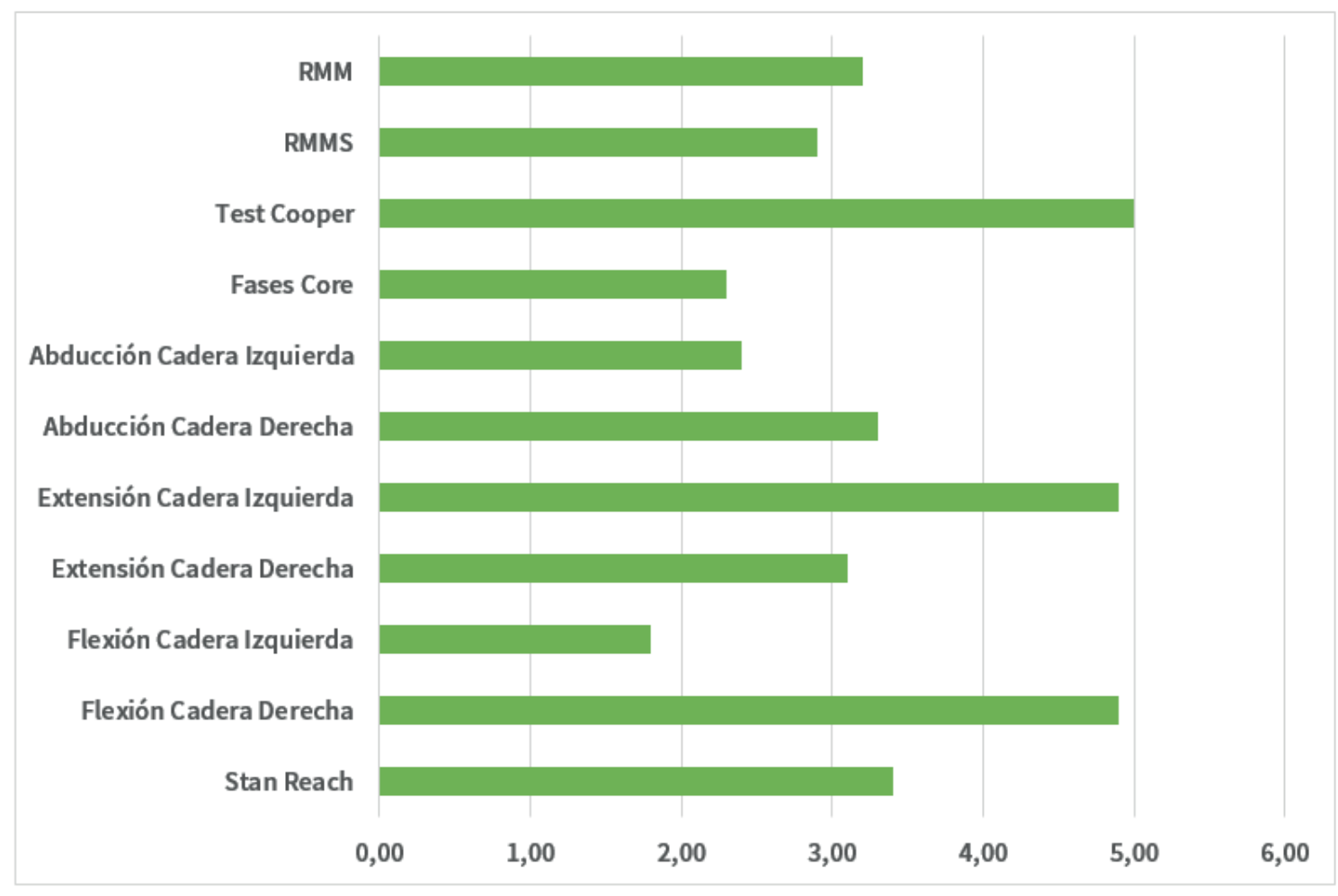

Fuente: elaboración propia (2017)

RMMI: Resistencia máxima

(1RM) Miembros inferiores,

RMMS: Resistencia máxima

(1RM) Miembros superiores.

El grafico indicó el tamaño del efecto con d de Cohen, con cambios grandes en todas las cualidades físicas, siendo más relevante la resistencia cardiovascular (d cohen 3,52), seguido de la flexión cadera derecha y extensión cadera izquierda (d cohen 3,41 y 3,42). Hubo valores un poco más bajos en el caso de la flexión de cadera izquierda ( $\mathrm{d}$ cohen 1,34$)$ y la fuerza muscular central evaluada con las fases del Core (d Cohen 1,79).

Grafico 2. Resistencia cardiovascular.

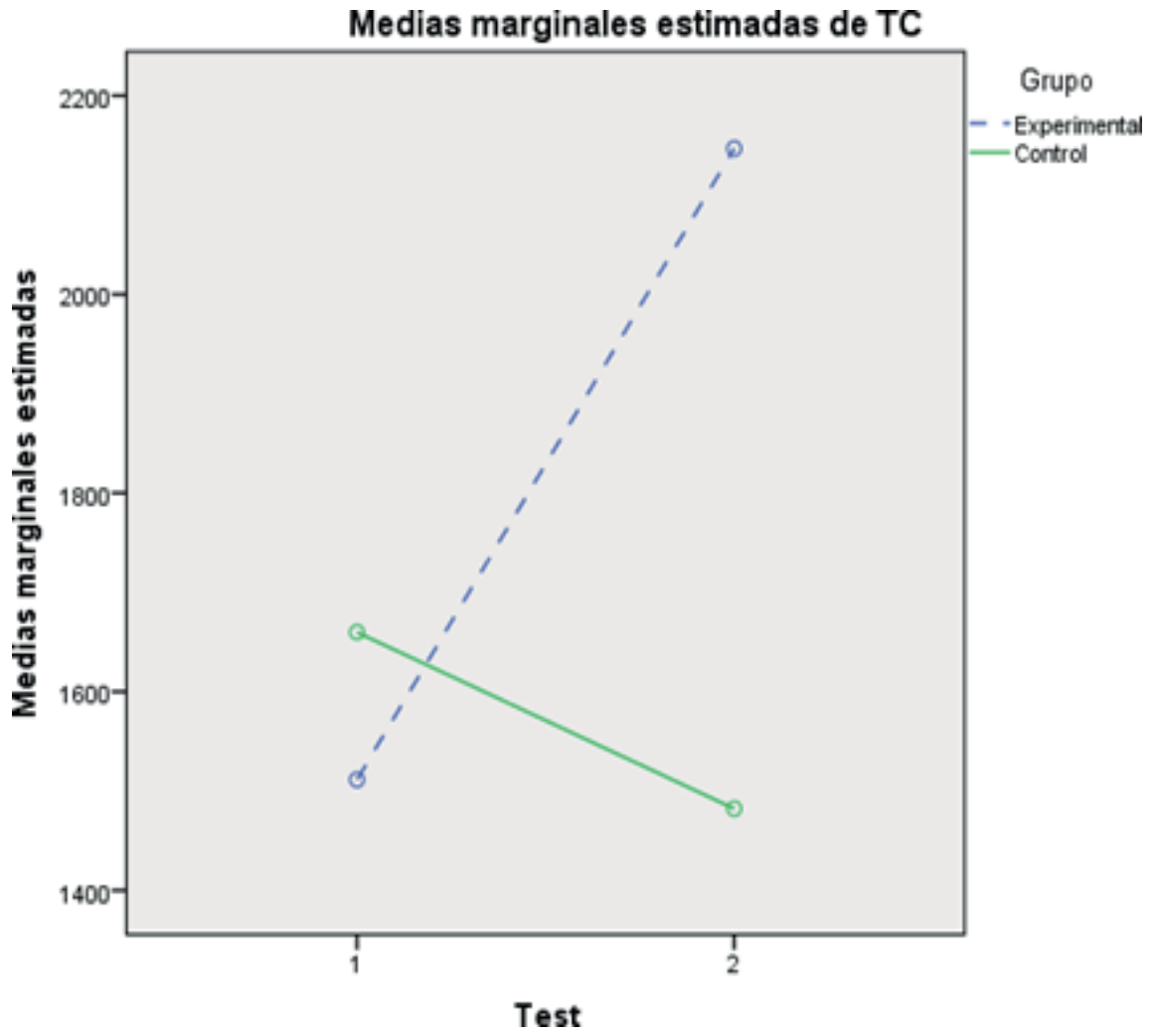

Fuente: elaboración propia (2017)

En el grafico 2 se identificó con claridad en línea verde continua el grupo control y en punteada azul el grupo experimental. El punto 1 es momento pre entrenamiento físico y el punto 2 post programa de entrenamiento, siendo incluso mejores los resultados en la resistencia cardiovascular del grupo control antes del programa de entrenamiento, posterior a este, la cualidad física resistencia cardiovascular, mejoró de manera importante en el grupo experimental con una línea en ascenso llegando hasta 2.146.80 metros recorridos, por otro lado, el grupo control redujo su resistencia cardiovascular en la evaluación post programa de entrenamiento y tiene una línea en descenso con reducción de hasta 177.57 metros.

\section{Grafico 3. Movilidad de cadera izquierda - Test Goniometría.}

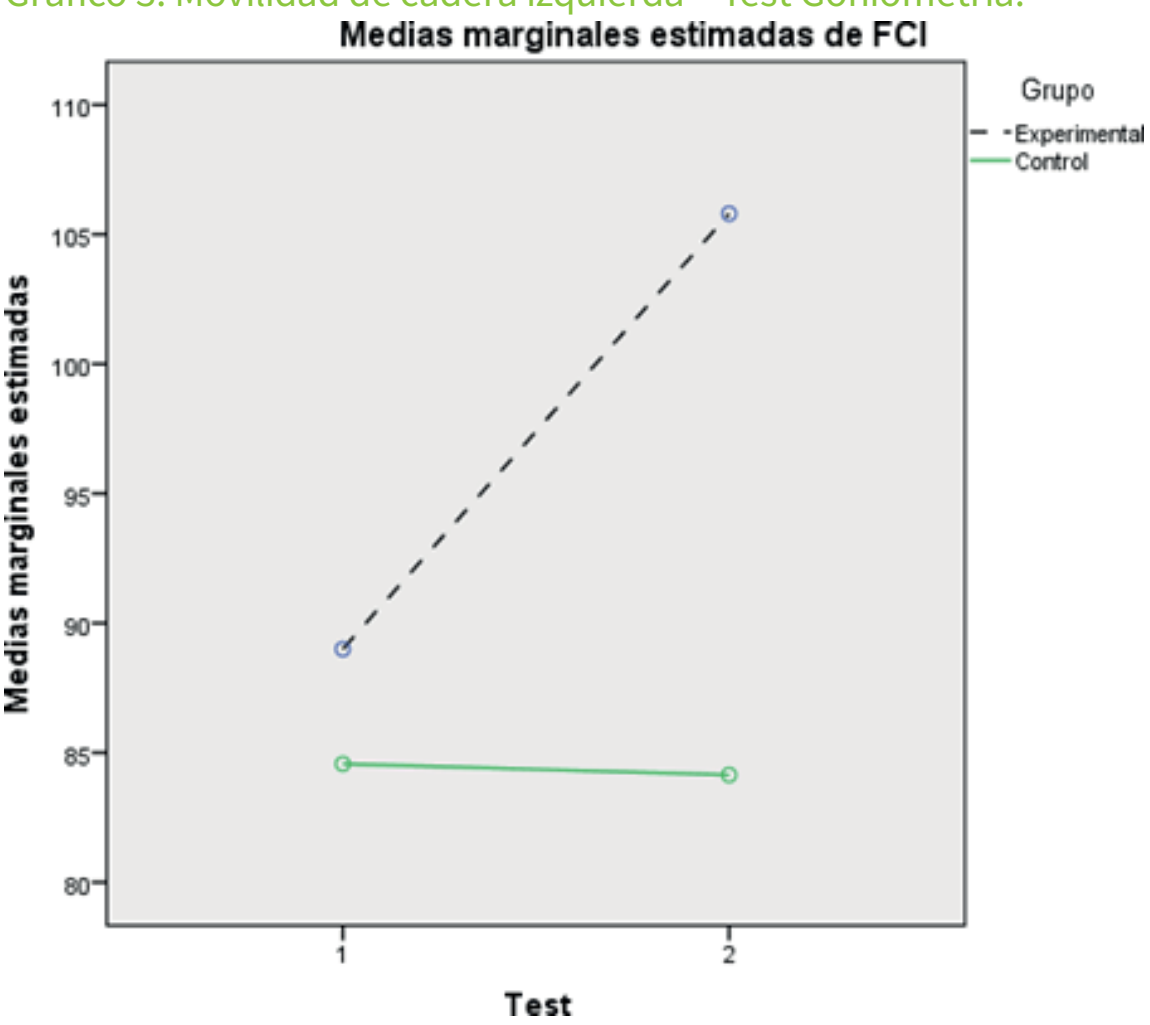

Fuente: elaboración propia (2017)

En el grafico 3 se idéntico en la flexión de cadera izquierda que los dos grupos iniciaron en rangos diferentes, indicando que el grupo experimental tuvo mejor rango de movimiento pre entrenamiento, mientras el control menor. El entrenamiento físico, potenció la movilidad de la cadera con una ganancia de hasta 105.8 grados, mientras el grupo control no tuvo cambios. 


\section{Discusión}

La actividad física para los estudiantes universitarios según los resultado de esta investigación es fundamental y se recomienda su aplicación en la formación profesional de Fisioterapia, debido a que dentro el bienestar físico un nivel de fitness adecuado, es clave para tener mejor desempeño en prácticas formativas y en la vida laboral, donde el fisioterapeuta es activo con el movimiento corporal humano, dirige ejercicio en diferentes niveles de complejidad y utiliza su cuerpo como herramienta de trabajo. Por su puesto, estos resultados, también aportan a los programas de bienestar Universitario, quienes más ala de dar opciones lúdicas en sus actividades, pueden implementar programas estructurados de entrenamiento los cuales en la población universitaria, tendrán grandes beneficios como se observó en esta investigación.

En otros estudios similares para aplicar programas de entrenamiento en estudiantes universitarios, se encontró un protocolo aplicado por 30 semanas, la duración del semestre de estudios, el cual ya era parte de la oferta académica del currículo universitario, con sesiones de 40 minutos 3 veces por semana (Sailors, 2011), a diferencia de este estudio, en donde fue necesario acortar los tiempos del programa de entrenamiento, debido a que el periodo académico tuvo una duración de 16 semanas, siendo necesario también intensificar las cargas para lograr cambios requeridos con sesiones de hasta 90 minutos 3 veces por semana. Uno de los productos propuestos de esta investigación fue implementar el programa de entrenamiento en el currículo académico con un curso de formación en entrenamiento físico para fisioterapeutas, lo cual se logró demostrando los efectos positivos del mismo. Otro estudio donde también se referenció un programa de entrenamiento, se encontró en estudiantes masculinos universitarios sedentarios y se midieron las cualidades físicas previas, pero los sujetos participaron en el programa de acondicionamiento físico durante 8 semanas (3 días por semana) con énfasis en mejorar la capacidad aeróbica, Fitness muscular, flexibilidad y composición corporal e incluso evaluar los conocimientos de fisiología. (Mehrabani, 2018). En ese estudio fue menor el tiempo de entrenamiento que el de esta investigación pero con las mismas cualidades físicas, que fundamentaron en su discusión la importancia de entrenar la capacidad cardiovascular, la fuerza y la flexibilidad en la población joven estudiantil, aunque en esta investigación se enfatizó en los estudiantes de Fisioterapia por los requerimientos para el actuar diario.

Identificando las cualidades físicas evaluadas y entrenadas se incluyó la flexibilidad, siendo esta la habilidad de una persona para moverse en el espacio sin restricción del sistema musculo esquelético, referida específicamente a articulaciones y esta tiene gran relación con la integridad articular y la capacidad de extensibilidad de los tejidos blandos (músculo, tendones, ligamentos, piel), (Pate, Oria, \& Pillsbury, 2012), por lo tanto, es la propiedad intrínseca de los tejidos corporales incluidos los músculos y tejido conectivo, que determina el rango de movimiento alcanzable sin lesiones en una articulación o grupo de articulaciones (Pate, Oria, \& Pillsbury, 2012), cualidad importante para el desempeño del Fisioterapeuta en la actividad laboral para la prevención de lesiones osteomusculares. Se encontró en estudios como el de Lopez-Bedoya y colaboradores (Lopez -Bedoya, 2013)quienes compararon el efecto de 3 técnicas de estiramiento (pasivo, FNP y activo), para la flexibilidad de cadera en 29 estudiantes sanos de último semestre de la Facultad de Actividad Física y Ciencias del Deporte de la Universidad de Granada en España, con un programa de entrenamiento de 9 semanas 2 sesiones por semana con duración de 40 minutos, entre calentamiento, fase central y vuelta a la calma. Encontraron cambios significativos $(P<0.5)$ entre el pre test y post test en la movilidad pasiva de flexión de cadera con el estiramiento pasivo, demostrando que este tipo de estiramiento es efectivo para los arcos de movimiento como se demostró en los rangos de amplitud articular de cadera en este estudio.

Sobre el entrenamiento de la fuerza, se tuvo en cuenta que los estudiantes aunque fueron sanos, igualmente fueron sedentarios y el entrenamiento muscular de pesos axiales o cargas máximas no fue el indicado para esta población, además de no ser el requerimiento funcional para las actividades del estudiante en Fisioterapia. En el programa de entrenamiento se enfatizó en la fuerza funcional por medio de la suspensión, ejercicios con pesos libres en diagonales de cadenas musculares, con sesiones que fueron intercaladas y combinadas con las otras cualidades físicas, dentro de las 12 semanas de entrenamiento. Se identificó que aunque la fuerza muscular para miembros superiores, inferiores y el Core no fue la de mejor puntuación, también fue estadísticamente significativa. El entrenamiento con suspensión también tuvo efectos en estudiantes jóvenes esquiadoras en un programa de seis semanas de ejercicio en donde igualmente se usaron ejercicios como squats, lunges laterales y frontales, press de pecho, curls de bíceps, press de tríceps, mountanin climbers, hamstring runner, hamstrings curl y press de cadera. En el estudio referido se encontró aumentos significativos en la fuerza, la coordinación de miembros superiores e inferiores, resistencia, estabilidad y balance (Curitianu, 2017). Por otro lado, el entrenamiento de la fuerza central o el Core, también fue uno de los objetivos de esta investigación, dada su importancia para la estabilidad del tronco, el control postural, y la fuerza global para las cadenas cinéticas de miembros superiores e inferiores, siendo necesaria para ejercicios de fuerza para cargar o movilizar pacientes, así como posturas prolongadas y ejercicios de repetición. En la evaluación pre test se encontró un pésimo nivel que da indicios para nuevas investigaciones que permitan relacionar este factor con alteraciones posturales, dolor lumbar crónico y mal alineación postural en los Fisioterapeutas y estudiantes. El entrenamiento en suspensión y funcional, fue el más utilizado para esta cualidad física, donde en este estudio según la tabla de evaluación de 8 fases del Core referido por el autor de textos deportivos Antonio Alba Berdeal, (Berdeal, 2006) se mejoró de 2.9 a 5 fases promedio, siendo esta una de las pruebas físicas de mayor dificultad. Según un estudio comparado con las actividades realizadas en esta investigación, se usaron igualmente ejercicios para el Core en suspensión como Roll Outs, BodySaw, y Knee-Tucks, aumentando otras comunes como planks en antebrazos y brazos y planks laterales. En el estudio comparado, se identificó gran actividad electromiográfica para músculos oblicuos abdominales y recto abdominal, (Cigliari, 2017) siendo estos ejercicios adecuados para su fuerza muscular, logrando una mejora en un componente del Core como los músculos en mención.

La resistencia cardiovascular, fue una cualidad física con grandes cambios, para el grupo experimental según los resultados del post test. Teniendo en cuenta que el programa de entrenamiento físico de esta investigación utilizó como método el circuito con entrenamiento funcional simulando actividades y movimientos propios de los fisioterapeutas con un énfasis cardiovascular, que al mismo tiempo permitió potenciar todas las cualidades físicas, siendo esta una actividad generalizada en las sesiones de entrenamiento, lo que demostró cambios importantes, así se comparó con el estudio de Al-Haliq, que utilizó el método de circuito para promover la mejora 
de los componentes del fitness en estudiantes universitarios, precisamente porque también lo justifica como uno de los métodos que incluye la potenciación de todas las cualidades físicas, y se asemejan los resultados porque el uso del circuito logró importantes mejoras en fuerza, resistencia cardiovascular y flexibilidad en todos los participantes (Hailg, 2015). El presente estudio identificó que en el VO2 Máximo, logró una mejora muy significativa con respecto a las distancias recorridas, mejorando 634.30 metros mientras en el grupo control se pudo observar una disminución de 177 metros. Esto se comparó con el estudio de Mayorga-Vega y colaboradores (Mayorga- Vega, 2013) quienes aplicaron un programa de circuito de entrenamiento en jóvenes escolares, durante 4 semanas con 14 sesiones. Encontraron mejoras importantes en la resistencia cardiovascular y el rendimiento muscular, aunque las pruebas de medición difirieron a las de este estudio por la edad de los participantes tomando la batería EUROFIT (Council of Europe Committee for the Development of Sport 1998), especialmente diseñado para edades escolares. El presente estudio tuvo más semanas y sesiones de entrenamiento de manera combinada ya que no todas fueron circuito funcional, debido a los énfasis requeridos para mejorar otras cualidades físicas, sin embargo, haciendo la conversión de las distancias recorridas por los participantes con el volumen máximo de oxígeno, se encontró en el grupo experimental un pretest de $29.55 \mathrm{ml} / \mathrm{kg}-\mathrm{l} / \mathrm{min}-1$ VO2 Max a $40.86 \mathrm{ml} / \mathrm{kg}-\mathrm{l} / \mathrm{min}-1$ VO2 Max, y el grupo control un pretest de 32.78 $\mathrm{ml} / \mathrm{kg}-\mathrm{l} / \mathrm{min}-1$ VO2 Max a $29.55 \mathrm{ml} / \mathrm{kg}-\mathrm{l} / \mathrm{min}-1$ VO2 Max. La mejora en resistencia cardiovascular del grupo experimental también se comparó con el estudio de Sonchan W y colaboradores (Sonchan, Moungmee, \& Sootmongkol, 2017), donde se aplicó entrenamiento en circuito por ocho semanas con variedad de ejercicios funcionales, en donde los participantes mejoraron valores de $46.46 \mathrm{ml} / \mathrm{kg}-\mathrm{l} / \mathrm{min}$ 1 a $55.09 \mathrm{ml} / \mathrm{kg}-\mathrm{l} / \mathrm{min}-1$, demostrando que otros jóvenes tuvieron mejores rangos de VO2 max en pretest que los de este estudio tuvieron valores mucho menores y por lo tanto la ganancia fue mayor que la del estudio en comparación. En estos resultados se identificó que el grupo control disminuyó sus valores de distancia recorrida, siendo superiores los valores pre test que el grupo experimental, esto pudo darse porque el grupo control incrementó su sedentarismo al no participar en ninguna otra actividad deportiva como requerimiento para el desarrollo de la investigación y se disminuyó su condición física incluyendo la resistencia cardiovascular de manera significativa, ya que las capacidades físicas solamente se logran estimular con entrenamiento, así se identificó en un estudio donde se concluyó que la relación dosis-respuesta entre la actividad física, el comportamiento sedentario y la calidad de vida relacionada con la salud es directamente proporcional (Wu, 2017)

La investigación presentó dificultades durante el desarrollo del programa de entrenamiento, debido a que los estudiantes entraron en periodo de vacaciones intersemestrales, lo que produjo la salida de la mayoría de ellos del programa de entrenamiento, quedando 7 estudiantes para el grupo control y 10 para el experimental, ya que son estudiantes que viven en municipios cercanos a la capital Pasto, salieron a sus lugares de procedencia, abandonando el programa. Esto permitió reflexionar sobre la falta de interés de los estudiantes universitarios para aprovechar espacios de ejercicio y entrenamiento dirigido, de manera voluntaria, ya que siempre debe estar relacionado con procesos académicos y cursos en formación más no fuera de este tiempo, lo que mostró la dificultad de esta población para adherirse de manera continuada al ejercicio, y por ello herramientas como la planificación del entrenamiento, permitiría tener organización en los ejercicios, medir los beneficios y darle orden al entrenamiento generando más participación de los jóvenes en las universidades.

\section{Agradecimientos:}

Al programa de Fisioterapia de la Universidad Mariana y los estudiantes que participaron en el estudio, también al docente Arsenio Hidalgo por su apoyo estadístico desde la Universidad de Nariño y al docente Henry Andrade por ser parte de este proyecto.

\section{Fuentes de financiación:}

El presente estudio fue financiado por el programa de Fisioterapia de la Universidad Mariana.

\section{Conflicto de intereses:}

Ninguno declarado por la autora.

\section{Referencias}

Anderson, S. P., \& Oakman, J. (2016). “Work-Related Musculoskeletal Disorders among Physical Therapists: A Systematic Review. Journal of Back and Musculoskeletal Rehabilitation, 29(3): 417-28.

BAE, Young-Hyeon, and Kyoung Sam MIN. . (2016). Associations between Work-Related Musculoskeletal Disorders, Quality of Life, and Workplace Stress in Physical Therapists. Industrial Health , 54(4): 34753.

Berdeal, A. (2006). Evaluacion y prescripcion de Acondicionamiento Fisico. Kinesis

chaparro, L. V. (2014). universisdad saludables a partir de los estilos de vida.

Cigliari, G. a. (2017). Core Muscle Activation in Suspension Training Exercises. Journal of Human Kinetics, 56(1): 61- 71.

Curitianu, L. (2017). Effescts Of six - Week TRX Training On Physical Skills InFamale Skier Students. The European Proceedings of Social \& Behavioural Sciences., 1456- 60.

Hagell, D. A. (October de 2013). Association For Young People's Health. Health: Update 2014, 11. Obtenido de http://www. youngpeopleshealth.org.uk/wp-content/uploads/2015/07/Youngpeoples-health-Update-2014.pdf.

Hailg, A. (5 de agosto de 2015). using the circuit Training Method to Promote The Physical Fitness Components of the Hashemite University Students. advance in physical Education, 170- 75.

Lopez -Bedoya, J. M.-S.-F.-V. (2013). "Effect of Theree Types of Flexibility Training on Active and Passive Hip Range of Motion". jornaul of sports Medicine and Physical Fitness, 53(3): 240- 47.

Mahecha, M. A. (2012). "Revision de la practica Profesional Fisioterapeutica y Otras Areas de La salud En Los Cuales ha Generado Responsabilidad Juridica Entre Los años 2000 y 2010.". Univesidad CES- UAM. Obtenido de http://bdigital.ces.edu.co:8080/repositorio/ bitstream/10946/1587/4/Revision_practica_profesional.pdf.

Mayorga- Vega, D. J. (2013). "Effects of a circuit Training Program on Muscular and Cardiovascular Endurance and their Maintenance in Schoolchildren.". Journal of human kinetics, 37(1): 153- 60. 


\section{Efectos de un programa de entrenamiento físico para estudiantes de Fisioterapia}

Mcphail, S. M. (2014). "Physical Activity and Health - Related Quality of Life among Physiotherapists: Across Sectional Survey in an Australian Hospital and Heart Service.". Journal of Occupational Medicine and Toxicology, 9(1): 1- 9. Obtenido de https://www.ncbi.nim.nih.gov/ pmc/articles/PMC3896696/pdf/1745- 6673-9-1.pdf.

Mehrabani, J. a. (2018). "Participation in Educational Physical Training, Program: Physical Fitness, Exercise Physiology Knowledge and Fitness Satisfaction in Inactive Male College Student.". Journal of Phyical Fitness, Medicine \& Treatment in sports 2(2). Obtenido de https://juniperpublishers.com/jpfmts/pdf/JPFMTS.MS.ID.555581.pdf.

Pate, R., Oria, M., \& Pillsbury, L. (2012). Fitness Measures and Health Outcomes in Youth. Washington D.C: National Academy of Sciences.

Peterson, N. E. (2018). "Sedentary Behavior and Physical Activity of Young Adult University Students." . Research in Nursing and Health., 41(1): 30- 38.
Sailors, M. H. (2011). "Exposing College Students to Exercise: The Training Interventions and Genetics of Exercise Response (TIGER) Study." . 59(1): 13-20.

Sonchan, W., Moungmee, P., \& Sootmongkol, A. (2017). "The Effects of a Circuit Training Program on Muscle Strength , Agility , Anaerobic Performance and Cardiovascular Endurance. Digital Open Science Index, 11(4), 176-179.

Wu, X. Y. (2017). "The Influence of Physical Activity, Sedentary Behavior on Health-Related Quality of Life among the General Population of Children and Adolescents: A Systematic Review." . PLoS ONE, 12(11): $1-29$. 